\title{
Body Area Networks (BANs) - An Overview with Smart Sensors based Telemedical Monitoring System
}

\author{
Umar Talha \\ Dept. of Telecom \\ Engg, \\ Sir Syed University of \\ Engg. \& Technology, \\ Karachi, Pakistan
}

\author{
Mir Asif \\ Dept. of Electronic \\ Engg \\ Sir Syed University of \\ Engg. \& Technology, \\ Karachi, Pakistan
}

\author{
Sheraz Mohani \\ Dept. of Electronic \\ Engg \\ lqra University, \\ Main Campus, \\ Karachi, Pakistan
}

\author{
Jahanzeb Ahmad \\ Bristol robotics lab, \\ University of the West \\ of England, United \\ Kingdom
}

\begin{abstract}
The wireless communication technology and its development have progressed a lot in various fields of study, but none so more than in the field of wireless networks. Among the various research areas and applications of this technology is BAN or Body Area Network, which can be defined as a miniature network consisting of sensors operating mainly on or around the human body. Technological advancements in devices with features like low cost, low size, ultra-low power consumption and emission, with more reliable communication expands the span of usage scenarios and applications especially in healthcare and monitoring. A health-care BAN application may comprise of sensors (wearable or implanted) and servers (personal and central). Such a network gives rise to two communication types categorized as Intra-BAN communication (sensors and personal device communication) and Inter-BAN (communication between servers). Classification of BANs on the basis of the device-operation environment can be divided in two main categories: wearable BANs and implanted BANs. These categories and their cooperation issues have particular importance since requirements of BANs change with the operating environment. This paper presents a comparison of wearable BANs with implanted BANs also covering the important issues mentioned, also identifying the differences between a normal Wireless Sensor Network and BAN. Furthermore, the paper also proposes human body communication to be utilized for BAN.
\end{abstract}

\section{General Terms}

Body Area Network (BAN), Wireless Sensor Networks.

\section{Keywords}

Telemedical health monitoring system, Wearable and implanted BANs.

\section{INTRODUCTION}

With the technical advancements and emergence of sophisticated semiconductor technologies, the modern age has seen the advent of smaller devices, these advancements have also enabled the development of so miniature and intelligent biomedical sensors that can easily be attached in or around the human body. On the other hand, the employment of wireless networks is becoming more and more extensive with the increase in the number of devices being able to communicate wirelessly. At present, the wireless communication is available with nano-size devices by these technologies. The blend of these two advancements, i.e. the wirelessly corresponding sensors inside or around the human body and the study of networks, sets the foundation of a new type of network: a Body Area Network or BAN. A BAN is simply a network of these advance inter and intra- communicating sensors implanted in or around the body [1]. These sensors may supply a central medical server or home station with the status of essential parameters like pulse rate, blood pressure and glucose level from near or inside the human body, thus enabling remote monitoring of patients.

Currently, IEEE 802.15 working group have established a task group, wireless body area networks (TG-WBAN), the workgroup will be responsible for developing the basic guidelines for using a small range wireless technology in fields of health related and consumer services [2]. Moreover, these networks are becoming more and more successful for sporting activities, healthcare systems and military services. BANs are now are considered as a key technology to prevent the occurrence of heart related issues like blockage of arteries which supply the heart muscles, and injuring of the heart muscle, monitoring the health status of patients with long lasting diseases, such as Diabetes and Asthma, Cardiology events where ECGs of long duration are required for therapy and most importantly in indicating early signs of a potential heart attacks.

BANs enable prolonged monitoring of patients under usual physiological states excluding restriction on them to carry out their normal activities. Many monitoring functions related to body were conventionally rarely monitored or if monitored were sampled by a significant period of time. For instance the cardio rhythm disturbances can be monitored by Holter monitors but only for offline processing, and not for providing a real-time feedback [3] [4].

\section{MEDICAL WIRELESS BAN}

A typical medical health monitoring wireless BAN may consist of the said small sized, ultra-low power consuming and emitting sensor(s) which may be wearable or implanted inside the body, a home station or expert home system which includes a remote transmitter and lastly a medical platform which receives the data to be reviewed by the doctor. For instance consider a wireless sensor pack installed on or in the body of a patient, this sensor may monitor and record some readings like the ECG, pulse rate or temperature etc. and then they are wirelessly transmitted by the on-board transmitter to the home station- which may be a PDA or a Laptop. It may include a home expert system, which may be a first hand signal modifier or an actuator- which may take some action on the basis of the sensor readings like the regulation of insulin for diabetics patients, The home station in conjunction with the home expert system sends the data to the remote medical platform, suppose a hospital which may include a data and application center for applications like storage of measured values, diagnosis on the basis of the input, activating alarm or simply presented to a doctor for review [5]. Inside a typical medical BAN the attached or in-body devices may be categorized into two types: sensors and actuators, functions of 
both the devices are already being distinguished. Figure 1 demonstrates an example of a typical medical BAN and the flow of data in it.

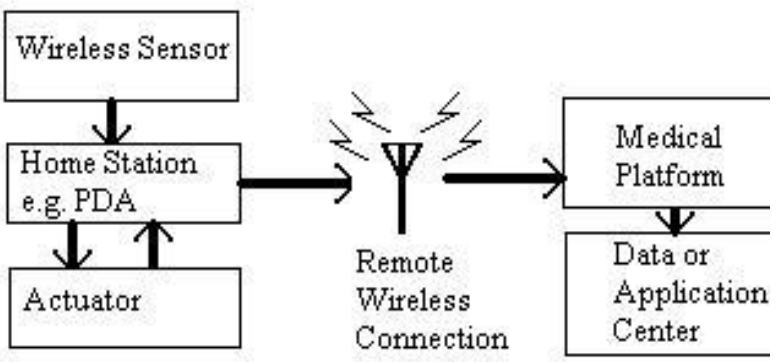

Figure 1 :Data flow in a typical medical BAN

The wireless BANs (WBANs) are different from other wireless sensor networks (WSN) and work groups in IEEE 802 committee mainly because of such medical and healthcare applications. The two said types of networks, the WBAN and WSN, obviously shares a lot in common like both are very limited in terms of available resources, such as the available battery, bandwidth, memory, various coexistence surroundings and energy efficiency. Table I demonstrates a comparison of the two types of networks in consideration, i.e. Typical health care WBAN sensor networks with common wireless sensor networks (WSN), both of which are primarily employed by low-rate Wireless Personal Area Network technologies. Comparison is made on the basis of features such as the sensors or actuators in use, reliability and dependability, networking type used and traffic etc. [6] [7].

\section{WEARABLE BANS}

BAN system with wearable sensors, incorporated into a telemedicine health monitoring system is a technology that enables a premature recognition of critical or abnormal conditions and helps in the avoidance of severe cost related to it in case of a failure [8] [9]. Wearable type BAN systems can be advantageous in prolonged monitoring of continuous administered conditions, rehabilitation and surgeries. Such systems, currently on hand, have the following limitations with them for the higher endorsement:

- Sensors and processors or servers are inconveniently wired

- Nodes (sensors) are not well incorporated

- Sharing of wireless channel by multiple nodes results in interference

- High data compilation and storage support is not available

A wearable type BAN used in health-monitoring is as such that the sensing device can be incorporated within the clothing of the person to be monitored [10]. However the system may be considered inappropriate with activities such as swimming, intensive training or even pro-longed computer assisted treatment [11] [12]. There have been lot of development in this particular field and the integration of radio modules, sensors and controllers on a chip, networking of low-sized wireless sensors ensures that these will be more and more suitable for a wider range of applications [13] [14] [15] [16]. Wireless channels are generally used in most such applications for the data transfer between sensors and servers, also the conventionally complex protocols such as Bluetooth and Wi-Fi are equally vastly used, which also consume more energy and power [17]. Sometimes when lengthy wearable observations are required such features, as described above, make their use inappropriate. Luckily, solutions which cater this with a great deal of more effectiveness are possible, solution like the ZigBee [17] [18]. ZigBee is considered by many as a uprising in the industrial WPANs, with it enabling short range communication possible with low power and rate, it also provides other effective features like meshing.

Many smart applications for home such as controlling gates, lighting and cameras are now using ZigBee, however healthcare application have not yet been beneficial from it and the employment rate of ZigBee in such applications is considerably low as compared to other counter parts, despite the fact that the amount of loss is much severe in such applications.

\section{IMPLANTED BANS}

Monitoring and diagnosis different conditions in health care have benefited greatly by medical implants, although it can still be considered as a very new concept. To name a few of the major accomplishment areas of such implants are cancer and heart disease detection and monitoring. Crucial data is sent to the doctor or the medical desk wirelessly with the help of these sensors and enable the doctor to prepare the strategy for therapy upon this important information.

With regards to size and weight of the sensors, medical implants have more strict limitations; therefore it is restricted in some important features like memory, power and processing specifications.

However, considering the fact that when replacing such sensors with surgery the consequences such as patient trauma and disorders and hefty cost associated with the operation, the amount of lifetime for an implant sensor should be as increased as reliably possible. Some other considerations are also very important such as managing energy resources for the low power devices used, for instance processor, transceiver and sensors. As any alien substance in the human body has to fight against the natural immune system present in human body the compatibility of the device implanted is very much essential. Regardless of the fact that the radio signals and networking are key features the surgeon locates the implant where it can supply him with the most important, appropriate information and also considering the ease of the patient, thus this location of the implant has a vital significance. Location of implant is another challenge. A medical implant will be located by surgeon to where it provides the best patient care and ease, with little consideration on the radio propagation and network [19].

The data rates observed in the communication of implanted sensors have a broad spectrum and the ranges generally vary from rates in low Kbps to high Mbps depending on the device using it. Generally the communication takes place among the device or sensor implanted and the servers (controller/ monitor) and this is also the set where the main data flow is observed, e.g. implanted neuroprosthesis, camera capsule and other sensors using nanotechnology (receiver/ transmitter). 
TABLE 1: HEALTH CARE WBANS COMPARED TO COMMON WIRELESS SENSOR NETWORK

\begin{tabular}{|c|c|c|}
\hline Feature & Health care WBAN s & Common WSNs \\
\hline Sensors/ actuators & $\begin{array}{l}\text { - } \quad \text { Uni-functional devices } \\
\text { Inside a smaller range the movement is fast } \\
\text { General lifetimes in days, less than a decade } \\
\text { for implanted sensors [ days }<\text { Life }<10 \text { years] } \\
\text { Works on the principle of quality and user } \\
\text { safety first }\end{array}$ & $\begin{array}{ll}\text { - } & \text { Devices are multi-functional } \\
\text { - } & \text { Larger range corresponds to slow movement } \\
& \text { lifetimes in months, also less than about a decade } \\
& \text { Sensitivity: cost driven }\end{array}$ \\
\hline Topology and link & $\begin{array}{l}\text { Generally star topology and small scaled } \\
\text { and must be robust as the body can be in } \\
\text { motion } \\
\text { - No redundant devices } \\
\text { - Deterministic node allocation }\end{array}$ & $\begin{array}{l}\text { - } \quad \text { Normally large scaled } \\
\text { - } \quad \text { Redundant devices are a common deploy } \\
\text { - }\end{array}$ \\
\hline Communication & $\begin{array}{l}\text { M:1 communication is observed } \\
\text { Periodic unidirectional RT dominant traffic } \\
\text { with priority given to burst }\end{array}$ & $\begin{array}{l}\text { M:1 or Point to point communication is generally } \\
\text { observed } \\
\text { - } \quad \text { Periodic and predominantly burst Traffic type } \\
\text { - } \quad \text { Both Uni/Bi- directional traffic is found }\end{array}$ \\
\hline Reliability and QoS & $\begin{array}{l}\text { Normally high security driven networks, giving } \\
\text { reliability with high and guaranteed QoS the } \\
\text { major share of consideration }\end{array}$ & $\begin{array}{l}\text { Comparatively lower security driven networks, reliability is } \\
\text { based upon redundancy, good QoS is anticipated but not } \\
\text { guaranteed. }\end{array}$ \\
\hline Band & $\begin{array}{l}\text { Uses ISM band, medical channel, generally } \\
\text { through body or on its outside }\end{array}$ & ISM band is used but with unknown obstruction \\
\hline
\end{tabular}

\section{RESEARCH WORK AND FUTURE APPLICATIONS}

BAN is a very extensive topic for research and applications, with application areas including human body communication, entertainment and various typical personal area network applications in conjunction with the health monitoring application discussed throughout the paper. In addition, extensive research and thorough insight are required in the areas of ultra-low power protocols and algorithms, coexistence and interference mitigation, cross-layer design, security and privacy, channel models, to name a few, in order to converge on the most suitable approaches for BAN.

\section{CONCLUSION}

BAN systems have the potential of revolutionizing the future in various fields especially in healthcare and entertainment. Home-based and remotely monitored patient remedy scenarios are anticipated to have BAN sensors in telemedical systems for higher efficiency and comfort. BAN telemedical systems can provide a key infrastructure for enabling enhanced and cheaper healthcare solution. Providing some remarkable advantages like early detection of certain conditions, prolonged monitoring, with the help of data mining extracting some important information and controlled treatment makes it beneficial to patients, doctors and community alike. . In conclusion the overview of BAN was given and its contrast with the other wireless sensor networks was investigated and a typical application of remote health monitoring system was also considered. Wearable and implanted both types of BANs were also explored with their components and issues.

\section{REFERENCES}

[1] K. Van Dam, S. Pitchers and M. Barnard, "Body Area Networks: Towards a wearable future", Proc. WWRF Kick off meeting, Germany, 6-7 March 2001.

[2] A.W. Astrin, "SG-BAN project authorization request draft", IEEE 15-06-0408-00-0ban, (in progress).

[3] August2009,http://www.nyp.org/health/electrocardiogra m-stresstest-holter.html.
[4] B. Lo and G. Z. Yang, "Key technical challenges and current implementations of body sensor networks", Proceedings of the 2nd International Workshop on Body Sensor Networks (BSN '05), pp. 1-5, April 2005.

[5] ShihHeng, Cheng and ChingYao, Huang, "Network Merging-Design strategies of an ultra low power and high reliability MAC", Project: IEEE P802.15 Working Group for Wireless Personal Area Networks (WPANs), March 2009 doc.: IEEE 802.15-09-0133-00-0006.

[6] B. Zhen, M. Patel, S. Lee and E. Won, "Body area networks (BAN) technical requirements", IEEE 802.15.BAN proposal (07-0867-03), 2007.

[7] L. Schwiebert, S.K.S. Gupta, and J. Weinmann, "Research challenges in wireless networks of biomedical sensors", ACM Conf. on Mobile Computing and Networks, pp. 151-165, 2001

[8] Istepanian RSH, Jovanov E, Zhang YT,"Guest Editorial Introduction to the Special Section on M-Health: Beyond Seamless Mobility and Global Wireless Health-Care Connectivity", IEEE Transactions on Information Technology in Biomedicine 2004, 8(4), pp, 405-414.

[9] Wearable Technology, Special Issue of the IEEE Engineering in Medicine and Biology Magazine 2003, 22(3).

[10] Park S, Jayaraman S, "Enhancing the Quality of Life Through Wearable Technology", IEEE Engineering in Medicine and Biology Magazine 2003, 22(3), pp, 41-48.

[11] Martin T., Jovanov E., Raskovic D., "Issues in Wearable Computing for Medical Monitoring Applications: A Case Study of a Wearable ECG Monitoring Device", Proceedings of The International Symposium on Wearable Computers ISWC Atlanta, Georgia 2000, pp, 43-50.

[12] Winters JM, Wang Y, Winters JM, "Wearable Sensors and Telerehabilitation: Integrating Intelligent Telerehabilitation Assistants With a Model for Optimizing Home Therapy", IEEE Engineering in Medicine and Biology Magazine, 22(3), pp, 56-65. 
[13] Center for Wireless Integrated Microsystems (WIMS) [http://www.wimserc.org/]

[14] Ghovanloo M, Najafi K, "A BiCMOS Wireless Stimulator Chip for Micromachined Stimulating Microprobes", Proceedings of the Second Joint EMBS/BMES Conference 2002, pp, 2113-2114.

[15] Otis BP, Rabaey JM, “A 300- $\mu \mathrm{W}$ 1.9-GHz CMOS Oscillator Utilizing Micromachined Resonators", IEEE Journal of Solid-State Circuits 2003, 38(7), pp, 12711274.
[16] Raskovic D, Martin T, Jovanov E, "Medical Monitoring Applications for Wearable Computing", The Computer Journal 2004, 47(4), pp, 495-504.

[17] R. Ashok, D. Agarwal, "Next-Generation wearable networks", IEEE Computer, Vol. 36, No. 11, November 2003, pp, 31-39

[18] ZigBee Alliance Document 02130, Network Layer Specification, July 2004

[19] M. Moh, B.J. Culpepper, and L. Dung, et al, "On data gathering protocols for in-body biomedical sensor networks," IEEE 2005 